\title{
On the stored and dissipated energies in heterogeneous rate-independent materials.
}

\author{
Application to a quasi-brittle energetic material under tensile loading.
}

the date of receipt and acceptance should be inserted later

\begin{abstract}
The present paper is the first part of a work that aims at building a dissipative model of microcrack friction in quasi-brittle energetic materials. The latter is viewed as an assembly of elementary cells containing the most salient features of the heterogeneous microstructure of an energetic material. It is intended here to build an analytical model describing the mechanical and energetic response of such an elementary cell under confined tension. This is achieved by applying a previously published theory that allows for the determination of the amount of dissipated and stored energies in heterogeneous dissipative structures containing microcracks and other dissipative components.
\end{abstract}

Keywords thermodynamics of irreversible processes $\cdot$ micromechanics $\cdot$ frictional cracks $\cdot$ plasticity dissipation

\section{Introduction}

The present paper is the second part of a work devoted to the evaluation of local dissipation in some highly heterogeneous quasi-brittle materials [1,2]. The field of energetic materials provides a good illustration of such a need. Explosives and solid propellants often display concrete-like microstructures, and deform in a very heterogeneous way. Under impact loading, heterogeneous heating occurs, and ignition (i.e. autonomous strongly exothermic chemical reaction, in other words a flame) may take place at the hottest locations, known as "hot spots" in the specialized literature [3]. It is thought that one of the most likely hot spot processes is caused by frictional microcracks $[4,5]$ in concrete-like energetic materials, such as the one whose microstructure is illustrated in Figure 1.

G. Vivier

CEA, DAM, Le Ripault, F-37260 Monts, France,

and LMT Cachan (UMR CNRS 8535), ENS Cachan/CNRS/Université de Paris 6/PRES Université Paris Sud

61 avenue du Président Wilson, F-94235 Cachan Cedex, France.

now at Saint-Gobain-Recherche, 39 quai Lucien-Lefranc

BP 135, F-93303 Aubervilliers Cedex.

E-mail: Guillaume.Vivier@saint-gobain.com

H. Trumel

CEA, DAM, Le Ripault, F-37260 Monts, France.

E-mail: herve.trumel@cea.fr

F. Hild

LMT Cachan (UMR CNRS 8535), ENS Cachan/CNRS/Université de Paris 6/PRES Université Paris Sud

61 avenue du Président Wilson, F-94235 Cachan Cedex, France.

E-mail: hild@lmt.ens-cachan.fr 


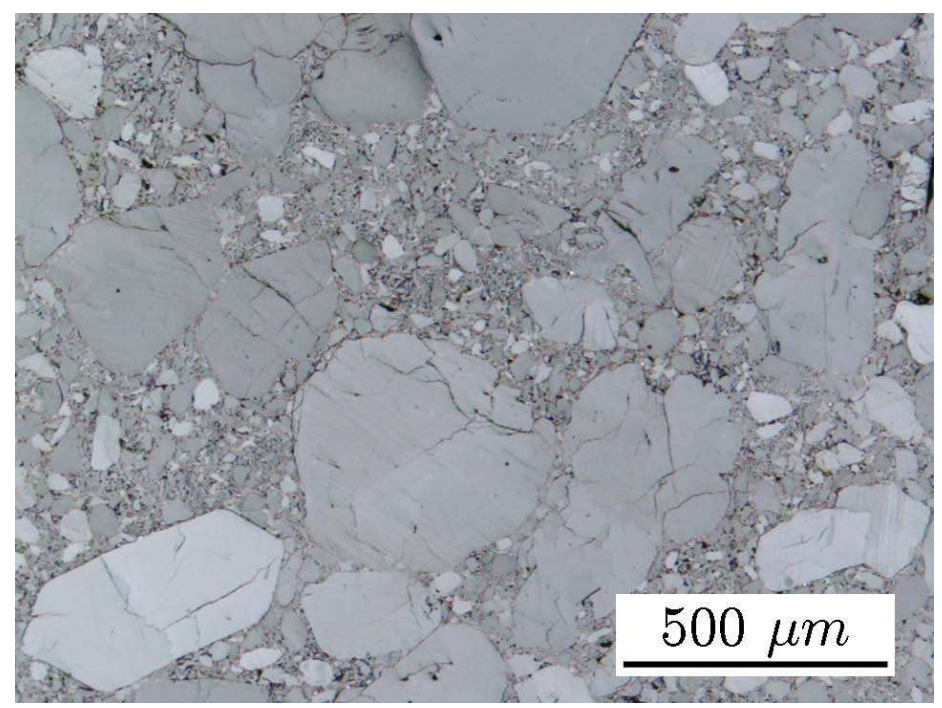

Fig. 1 A typical concrete-like microstructure for an energetic material [?].

Macroscopic ignition is the result of a nucleation, growth and coalescence of thermochemical processes. The very first events (i.e. nucleation), prior to any chemical reaction, are triggered by the competition between local dissipation (heat sources) and small scale thermal conduction (heat losses). It is thus crucial to determine the (intrinsic) dissipation as accurately as possible, and at the right scale. The problem consists in describing individual hot spots first, then the whole hot spot population. For this purpose, it is natural to consider the macroscopic material as an assembly of individual cells, each one possibly containing a single hot spot. Given the concrete-like nature of the material at stake (see Figure 1), the pioneering work of Andrieux et al. [6] can be seen as a good starting point. Aiming at designing a thermodynamically-based scale transition approach for concrete-like and rock-like materials, they considered the microcracked material as an aggregate of elementary cells. Each cell was assumed to contain a single microcrack embedded in a homogeneous elastic matrix, and submitted to the overall load at its boundaries.

This approach is attractive in that, in the dilute crack density limit, it allows one to employ fracture mechanics tools to derive micro-fields from the geometry of each crack, the elastic constants of the uncracked material (the matrix), and the macroscopic load. However, given the microstructure illustrated in Figure 1, this picture is clearly oversimplified, and must be adapted to account for strongly heterogeneous materials. Many energetic materials are polymers filled with more than $80 \mathrm{vol} \%$ crystals. In order to reach such high volume fraction, broad crystal size distributions are used, and the resulting microstructure can be viewed as large grains embedded in a composite matrix, with large grains volume fractions of the order of $50 \%$. The matrix, due to its polymer content, is generally much softer than the large grains. Energetic crystals are often brittle, and tend to develop transgranular cracks, especially under dynamic loading.

Therefore, the individual cells cannot be seen as a small microcrack embedded in a homogeneous elastic matrix , and must be replaced by a truly heterogeneous system. Moreover, given the large filler volume fractions to be considered, the assumption of separation of scales cannot hold either. Then, the material is seen as an aggregate of highly heterogeneous individual cells, submitted to a so-called mesoscopic load, supposed locally uniform on the cell boundaries, but allowed to fluctuate from cell to cell. According to this picture, a two-step scale transition is envisaged. In a first "micro-meso" step, a generic cell model is built using the effective moduli $[7,8]$ scale transition approach. The second "meso-macro" step uses the generic cell model as a black box in a statistical averaging process to derive the macroscopic model.

A very careful post-mortem examination of several samples showed that some of them contained small scale (of the order of 10 micrometers) molten areas. This is for example the case in a sample loaded quasistatically under a modest confinement of $20 \mathrm{MPa}$ [9]. This indicates that some of the dissipative processes are very fast, overcoming small scale thermal conduction, even when the overall load is slow. This was somewhat confirmed by (unpublished) acoustic emission experiments. It was thus decided to treat damage growth as a quasi-instantaneous process at the mesoscopic scale, to be dealt with in the meso-macro scale transition step, and to disregard trans-granular crack propagation. 
The present paper is concerned with the problem of elementary cell model derivation. It uses the general thermodynamically-based framework established previously [1]. The latter authors showed that the microstress field can be decomposed using a virtually unloaded intermediate state, in which residual self-balanced stresses and stored energy are defined. The concept of stored energy is of the same nature as the one defined by Taylor and Quinney [10] ("cold work energy" in their terminology and that of Aravas et al. [11]) in their famous study of the thermodynamics of strain hardening of metals, although the exact nature of the dissipative processes are not the same as theirs in the present case. This thermodynamical approach allows one to derive overall internal variables from microscopic fields, and ultimately to relate dissipated and stored energies to the overall load. Moreover, it is the authors' viewpoint that the stored energy is not only important in that it is not dissipated during loading, but also in that it can be released and dissipated during unloading and/or reverse loading.

However, the approach depends heavily on the cell and load geometry. The strong heterogeneity of the cell induces highly variable micro-fields, and a general solution of the cell problem cannot be obtained easily. Therefore, an approximate solution is sought, by considering a simplification of the local kinematics. This must be done very carefully, by checking the validity of the kinematic assumptions in a numerical fashion. In order to assess the feasibility of this approach, the present paper is restricted to the simple geometrical case of confined tensile loading, and should be supplemented by the more involved case of confined shear loading in a forthcoming paper.

Section 2 recalls the theoretical background of the previous paper [1], and extends it to the displacementbased approach. Stress and strain decompositions are then recalled, allowing some macroscopic mechanical and energetic quantities to be defined. Section 3 provides the method for determining the micro-mechanical fields, and Section 4 derives the overall resulting model for the stress-based case. In particular, this section shows how the virtually unloaded state can be reached using an internal stress as a control parameter, revealing to be a very useful and convenient modelling tool. It is interesting to examine the dual displacement-driven case, which is the purpose of Section 5. However, this approach is more involved, and the resulting overall cell model will be derived in a more approximate fashion. Some final comments are given to conclude the paper.

\section{Theoretical background}

Since this study is concerned with the development of a generic cell model in view of considering any representative volume element as a cell assembly, the following denominations are used. The homogenized behavior of a cell cannot be qualified as "macroscopic," and will therefore be referred to as "overall" or "mesoscopic" in the sequel.

\subsection{The effective moduli approach: Overall stresses and strains}

Let us consider a domain $\Omega$, bounded by its external surface $\Phi$, containing perfectly bonded elasto-plastic zones, closed and open cracks, and otherwise made of several perfectly bonded elastic phases. For the stressbased approach, the external boundary is submitted to a uniform overall traction

$$
\Sigma . \nu=\sigma . \nu \quad \text { on } \quad \Phi
$$

In this expression, $\boldsymbol{\Sigma}$ is the mesoscopic (overall) stress tensor, $\boldsymbol{\sigma}$ the microscopic stress tensor, $\boldsymbol{\nu}$ the outward unit vector, and bold symbols are used for vectors and tensors. In the absence of inertia and body forces, the following relationship applies

$$
\boldsymbol{\Sigma}=\frac{1}{V} \int_{\Omega} \boldsymbol{\sigma}(\mathbf{x}) d V
$$

where $V$ is the volume of domain $\Omega$, and $\mathbf{x}$ is the position vector of any material point of $\Omega$. Use is then made of the macro-homogeneity relationship $[7,8]$

$$
\boldsymbol{\Sigma}: \mathbf{E}=\frac{1}{V} \int_{\Phi}(\boldsymbol{\sigma}(\mathbf{x}) \cdot \boldsymbol{\nu}) \cdot \mathbf{u}(\mathbf{x}) d S
$$


to obtain the macroscopic strain $\mathbf{E}$

$$
\mathbf{E}=\frac{1}{V} \int_{\Phi} \frac{1}{2}(\mathbf{u}(\mathbf{x}) \otimes \boldsymbol{\nu}+\boldsymbol{\nu} \otimes \mathbf{u}(\mathbf{x})) d S=\frac{1}{V} \int_{\Phi} \mathbf{u}(\mathbf{x}) \otimes \boldsymbol{\nu} d S
$$

where the symbol $\otimes$ denotes the symmetrized tensorial product. This expression only uses boundary values, and the overall strain $\mathbf{E}$ can also be expressed in terms of internal fields as follows

$$
\mathbf{E}=\frac{1}{V} \int_{\Omega} \boldsymbol{\epsilon}(\mathbf{x}) d V+\frac{1}{V} \int_{\Gamma} \mathbf{u}(\mathbf{x}) \otimes \mathbf{n} d S
$$

where $\boldsymbol{\epsilon}(\mathbf{x})$ denotes the microscopic strain tensor, defined everywhere except on crack faces, $\Gamma$ the crack faces collectively, and $\mathbf{n}$ their outward normal vector (thus pointing from one face towards the other).

For the displacement-based approach, the external boundary $\Phi$ is submitted to a linear overall displacement

$$
\mathbf{u}(\mathbf{x})=\mathbf{E} \cdot \mathbf{x} \quad \text { on } \quad \Phi
$$

It is straightforward to show that Equations (4) and (5) are obtained directly from Equation (6). From the macro-homogeneity relationship (3), Equation (2) is recovered, and it can be shown that the macroscopic stresses are also defined in terms of boundary quantities as

$$
\boldsymbol{\Sigma}=\frac{1}{V} \int_{\Gamma}(\boldsymbol{\sigma}(\mathbf{x}) \cdot \boldsymbol{\nu}) \otimes \mathbf{x} d S
$$

2.2 Virtual paths. Stress and strain decompositions

Let us consider the overall stress-strain diagram of Figure 2. Using the superposition principle ${ }^{1}$, the real stress state at point $\mathrm{B}$ is decomposed as

$$
\begin{gathered}
\boldsymbol{\sigma}=\boldsymbol{\sigma}^{r e f}+\boldsymbol{\sigma}^{d}+\boldsymbol{\sigma}^{i} \\
\mathbf{u}=\mathbf{u}^{r e f}+\mathbf{u}^{d}+\mathbf{u}^{i}
\end{gathered}
$$

by using virtual (i.e. "thought experiments") unloading elastic paths. The stress field $\boldsymbol{\sigma}^{d}+\boldsymbol{\sigma}^{i}$ is obtained by unloading from $\mathbf{B}$, while freezing all dissipative processes and crack face displacements, down to point $\mathbf{C}$. The stress field $\boldsymbol{\sigma}^{d}$ is obtained by unloading from $\mathbf{B}$, while freezing dissipative processes but not crack face displacements, down to point $\mathbf{D}$. The fields $\mathbf{u}, \mathbf{u}^{r e f}, \mathbf{u}^{d}$ and $\mathbf{u}^{i}$ are the corresponding displacements. Note that since no external stress is applied to the cell at virtually unloaded points $\mathbf{C}$ and $\mathbf{D}, \boldsymbol{\sigma}^{d}$ and $\boldsymbol{\sigma}^{i}$ are self-balancing fields. Hence

$$
\begin{gathered}
\boldsymbol{\Sigma}=\frac{1}{V} \int_{\Omega} \boldsymbol{\sigma}^{r e f} d V \\
\frac{1}{V} \int_{\Omega} \boldsymbol{\sigma}^{d} d V=\mathbf{0} \\
\frac{1}{V} \int_{\Omega} \boldsymbol{\sigma}^{i} d V=\mathbf{0}
\end{gathered}
$$

The corresponding strain fields are defined as

$$
\epsilon^{a} \equiv \mathcal{C}^{-1}: \sigma^{a}
$$

where $\mathcal{C}$ is the local elastic (fourth order) stiffness tensor, the colon stands for the double contraction product, and ${ }^{a}$ is any of the ${ }^{r e f},{ }^{d}$, or $^{i}$ superscripts defined above. It is then possible to show that the overall strain tensor $\mathbf{E}$ may be decomposed into

$$
\boldsymbol{E}=\boldsymbol{E}^{r e f}+\boldsymbol{E}^{d}+\boldsymbol{E}^{i}
$$

\footnotetext{
${ }^{1}$ All theoretical developments are detailed in Ref. [1].
} 


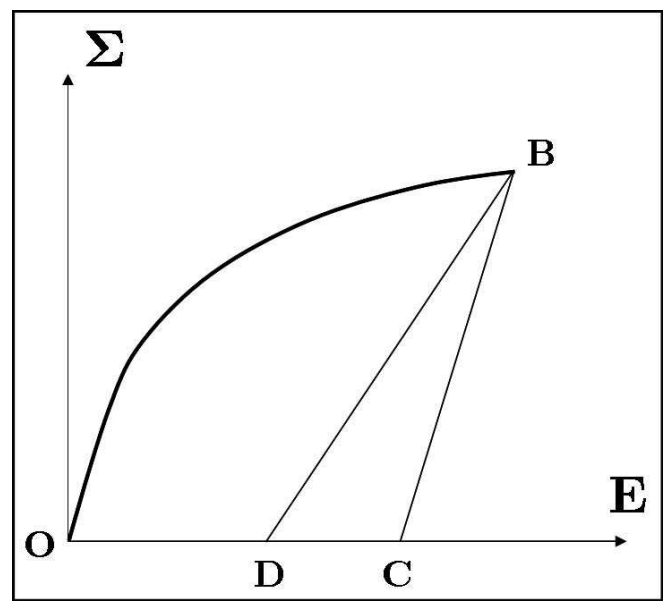

Fig. 2 Virtual thermodynamic paths used to build the components of the free energy.

where

$$
\begin{array}{r}
\boldsymbol{E}^{r e f}=\frac{1}{V} \int_{\Omega} \epsilon^{r e f} d V \\
\boldsymbol{E}^{d}=\frac{1}{V} \int_{\Omega} \epsilon^{d} d V+\int_{\Gamma} \boldsymbol{u}^{d} \otimes \mathbf{n} d S \\
\boldsymbol{E}^{i}=\frac{1}{V} \int_{\Omega} \epsilon^{i} d V+\int_{\Gamma} \boldsymbol{u}^{i} \otimes \mathbf{n} d S
\end{array}
$$

In these expressions, it must be stressed that $\mathbf{u}^{\text {ref }}$ is continuous everywhere, $\mathbf{u}^{d}$ is continuous everywhere except on open crack surfaces, and $\mathbf{u}^{i}$ is continuous everywhere except on all crack surfaces.

\subsection{Energies}

With those notations and definitions, the overall free energy $\Psi$ reads $[11,1]$

$$
\Psi=\frac{1}{V} \int_{\Omega} \boldsymbol{\sigma}: \mathcal{C}^{-1}: \boldsymbol{\sigma} d V
$$

can be shown to decompose into

$$
\Psi=W^{\star}+W^{i}
$$

with

$$
\begin{array}{r}
W^{\star}=\frac{1}{V} \int_{\Omega} \frac{1}{2}\left(\boldsymbol{\sigma}^{r e f}+\boldsymbol{\sigma}^{d}\right): \mathcal{C}^{-1}:\left(\boldsymbol{\sigma}^{r e f}+\boldsymbol{\sigma}^{d}\right) d V \\
W^{i}=\frac{1}{V} \int_{\Omega} \frac{1}{2} \boldsymbol{\sigma}^{i}: \mathcal{C}^{-1}: \boldsymbol{\sigma}^{i} d V
\end{array}
$$

such that the overall free energy $\Psi$ is the sum of recoverable elastic and stored energies $W^{\star}$ and $W^{i}$, respectively. They are evaluated independently at points $\mathbf{B}$ and $\mathbf{C}$, respectively. As shown in Ref. [1], the virtual path OC is followed by applying an internal stress on the transgranular crack; then, the virtual loading path CB is prescribed by increasing the external stress until suitable boundary conditions are met at the transgranular crack surface. Finally, according to the (isothermal) Clausius-Duhem inequality, the dissipation is given by [12]

$$
\mathcal{D}=\boldsymbol{\Sigma}: \dot{\boldsymbol{E}}-\dot{\Psi}=\boldsymbol{\Sigma}: \dot{\boldsymbol{E}}^{i}-\dot{W}^{i}
$$




\section{Local fields and overall elasticity: An approximation}

In principle, the dissipation can be evaluated if the above-mentioned microfields are known. For the complex material of Figure 1, an accurate description of microfields can only be achieved numerically. However, in the context of a future cell assembly, this would require large computational capabilities, and an approximate analytical approach is preferred. Hence, the elementary cell behavior will be sought in a plane strain twodimensional simplified way.

\subsection{The unit cell}

¿From a geometrical standpoint, we will consider a square elementary cell of length and width $L$, consisting of a rectangular inclusion (a large grain) of length $\ell_{x}$ and width $\ell_{y}$ embedded in a homogeneous matrix (thus neglecting the internal details of the latter), as illustrated in Figure 3. The third dimension of the cell will be denoted by $\ell_{z}$ in the sequel. This implies that some effects are neglected, and particularly direct grain contact and Hertz-like cracking. Although very simplified, this geometrical arrangement potentially contains the most salient physical processes of material deformation, among which unilateral contact and frictional sliding on both debond and transgranular cracks. The present paper is devoted to describing the unilateral effect and frictional sliding of inclusion-matrix debond cracks. This can be done under predominant confined overall tension with respect to transgranular opening direction, and has the advantage of allowing for a quasiunidimensional description of microfields. The case of frictional sliding of the transgranular crack will be examined under predominant overall shear loading in a forthcoming paper. The class of materials at stake is

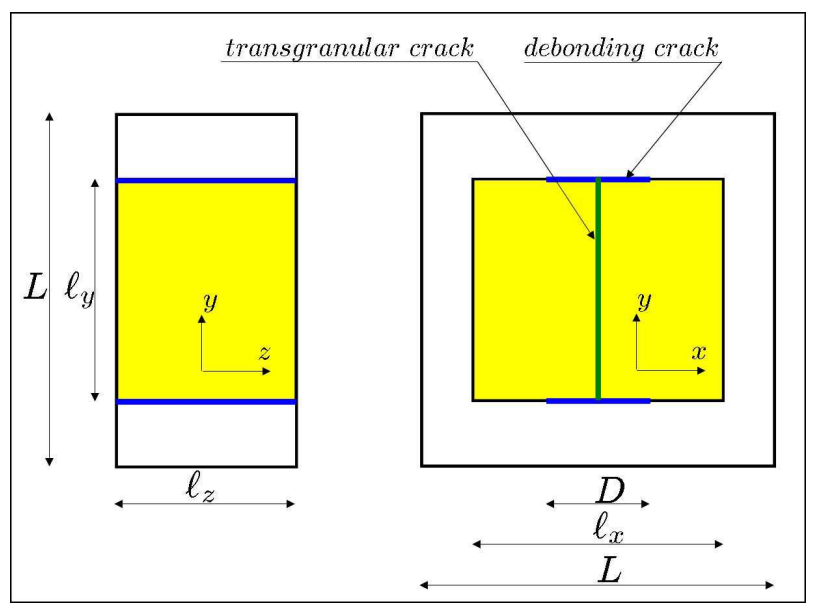

Fig. 3 The two-dimensional unit cell.

characterized by a high filler volume fraction, accounted for by taking $\alpha_{x} \equiv \frac{\ell_{x}}{L}=\alpha_{y} \equiv \frac{\ell_{y}}{L}=0.8$. This corresponds to a surface fraction of $64 \%$, and would achieve $50 \%$ in the three-dimensional case. In order to remain representative of an elastomer-bound material, $K_{I}=10 \mathrm{GPa}$ and $K_{M}=1 \mathrm{GPa}$ and taken for Young's moduli of the inclusion and the matrix respectively, and a common value of 0.3 is considered for Poisson's ratios of both constituents. Finally, the inclusion-matrix friction coefficient is taken to be $f=0.5$.

\subsection{Approximation of local fields}

The elastic response of the virgin elementary cell reads

$$
\mathbf{E}^{\text {ref }}=\mathbf{S}^{\mathrm{eq}} \boldsymbol{\Sigma}
$$

For the present case of confined tension, the response of the above-described elementary cell will be considered under restricted loads. In the reference frame of the cell (Figure 3), tractions or displacements normal 
to the cell boundaries will only be considered, and the macroscopic stresses and strains are both diagonal in Equation (21).

The cell is divided into three parts, namely, the longitudinal part of the matrix, the transverse part of the matrix, and the inclusion, respectively labelled $M l, M t$ and $I$ in the following. As illustrated in Figure 4, the approximate local fields are uniform in each part. In this fashion, the virgin cell may be considered as an assembly of springs as illustrated in Figure 5. In the following, tensile loads in the $x$ (longitudinal) direction will be considered, together with compressive confining stresses in the $y$ (lateral) direction. The local fields are also assumed diagonal in the cell reference frame, and the normal tractions continuous across internal interfaces (Figure 4). Tangential tractions may not be continuous across internal interfaces, although overall equilibrium is preserved, as shown below.

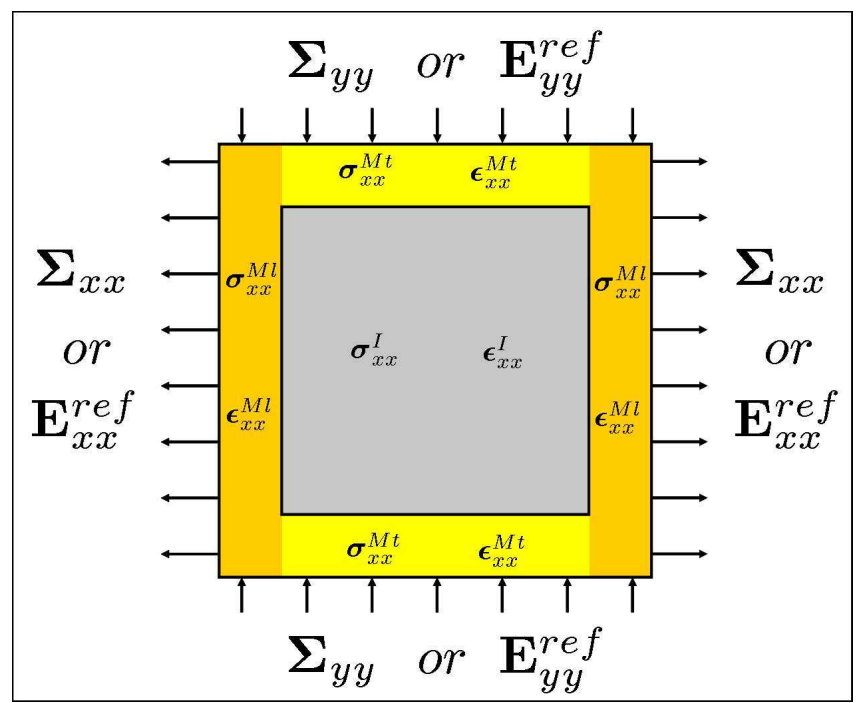

Fig. 4 Cell partition and approximations on local fields.

\subsection{Overall elastic response}

The local fields are considered to be uniform by parts. In this framework, the cell is represented as a spring assembly, as depicted in Figure 5, in the same spirit as in the simple examples of Ref. [1]. Then, the overall elastic response is expressed in the following Voigt-like orthotropic form

$$
\mathbf{E}^{\text {ref }}=\left(\begin{array}{c}
E_{x x}^{r e f} \\
E_{y y}^{r e f} \\
E_{x y}^{r e f}
\end{array}\right) ;\left(\mathbf{K}^{e q}\right)^{-1}=\mathbf{S}^{\mathbf{e q}}=\left(\begin{array}{ccc}
S_{x x}^{e q} & S_{x y}^{e q} & S_{x z}^{e q} \\
S_{x y}^{e q} & S_{y y}^{e q} & S_{y z}^{e q} \\
S_{x z}^{e q} & S_{y z}^{e q} & S_{z z}^{e q}
\end{array}\right) ; \boldsymbol{\Sigma}=\left(\begin{array}{c}
\Sigma_{x x} \\
\Sigma_{y y} \\
\Sigma_{z z}
\end{array}\right)
$$

where the components of the orthotropic stiffness tensor $\mathbf{S}^{\mathbf{e q}}$ are given by

$$
\begin{gathered}
S_{x x}^{e q}=\frac{1}{K_{x x}^{e q}} ; S_{y y}^{e q}=\frac{1}{K_{y y}^{e q}} ; S_{z z}^{e q}=\frac{1}{K_{z z}^{e q}} \\
S_{x y}^{e q}=\frac{-\nu_{x y}^{e q}}{K_{x x}^{e q}} ; S_{x z}^{e q}=\frac{-\nu_{z x}^{e q}}{K_{z z}^{e q}} ; S_{y z}^{e q}=\frac{-\nu_{z y}}{K_{z z}^{e q}} \\
\nu_{x y}=\nu_{z x}=\nu_{z y}=\nu_{I}=\nu_{M}=\nu
\end{gathered}
$$

Then, Young's moduli $K_{x x}^{e q}$ and $K_{y y}^{e q}$ are given by

$$
\begin{gathered}
K_{x x}^{e q}=\frac{1}{\frac{1-\alpha_{x}}{K_{M}}+\frac{\alpha_{x}}{K_{x x}^{\prime}}} ; K_{y y}^{e q}=\frac{1}{\frac{1-\alpha_{y}}{K_{M}}+\frac{\alpha_{y}}{K_{y y}^{\prime}}} \\
K_{x x}^{\prime}=\left(1-\alpha_{y}\right) K_{M}+\alpha_{y} K_{I} ; K_{y y}^{\prime}=\left(1-\alpha_{x}\right) K_{M}+\alpha_{x} K_{I}
\end{gathered}
$$




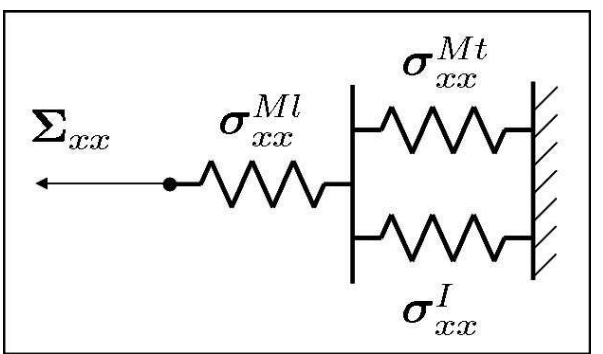

Fig. 5 Spring assembly to build the simplified overall elastic response of the elementary cell.

whereas $K_{z z}^{e q}$ modulus need not be specified in the present plane strain context. As will be seen below, although highly simplified, this description will reveal sufficient for the present purpose. The results of the theory will be systematically compared to numerical simulations using the finite element code ABAQUS, after having checked numerical convergence versus mesh size.

\section{Stress-driven approach}

4.1 The elastic undamaged case

Besides providing the response of the elastic undamaged cell during the appropriate virtual loading path, the aim of the present and the next sections is to determine whether a quasi-unidimensional representation of local fields is appropriate or not. In the present case, the cell is only loaded by a longitudinal external stress $\Sigma_{x x}^{r e f}$, and the overall longitudinal strain $E_{x x}^{r e f}$ and the elastic energy $W^{r e f}$ are sought. Using the above-described approximations, the local stresses in the inclusion and the matrix respectively read

$$
\begin{aligned}
\sigma_{I x}^{r e f}(x) & =\frac{K_{I}}{K_{x x}^{\prime}} \Sigma_{x x} \\
\sigma_{M x}^{r e f} & =\frac{K_{M}}{K_{x x}^{\prime}} \Sigma_{x x}
\end{aligned}
$$

whereas the longitudinal part of the matrix is submitted to the macroscopic stress $\Sigma_{x x}$. In this expression, the subscripts I and $\mathrm{M}$ denote the inclusion and the lateral part of the matrix respectively. Using Equation (2), the longitudinal response reads

$$
\Sigma_{x x}=K_{x x}^{e q} E_{x x}^{r e f}
$$

where $K_{x x}^{e q}$ and $K_{x x}^{\prime}$ are given by Equations (24) and (25). With the above-mentioned values of material constants, $K_{x x}^{e q}=3.36 \mathrm{GPa}$ and $K_{x x}^{\prime}=8.2 \mathrm{GPa}$ are obtained. A useful result, to be used in the following, is the average (normal) stress $\sigma_{x x}^{N}$ at the transgranular crack location of the inclusion

$$
\sigma_{x x}^{N}=\frac{K_{I}}{K_{x x}^{\prime}} \Sigma_{x x}
$$

The strain energy of the cell is given by

$$
W^{r e f}=\frac{1}{2} \frac{\Sigma_{x x}^{2}}{K_{x x}^{e q}}
$$

Those results compare favorably to numerical ones, as shown in Figure 6. Hence, the quasi-unidimensional representation of local fields appears adequate to capture the essential features of the overall elastic behavior of the undamaged cell. 


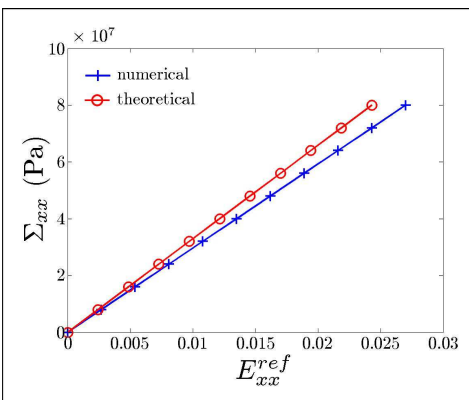

(a) mechanical response

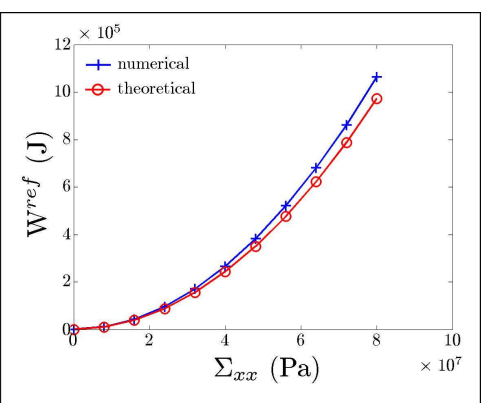

(b) energetic response

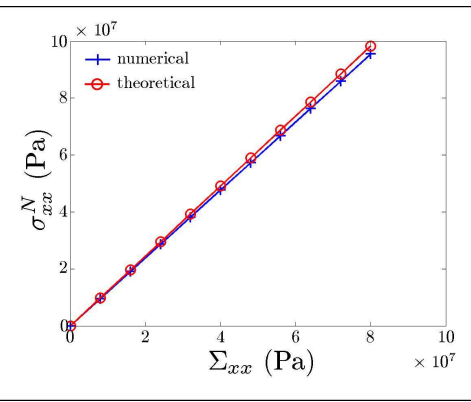

(c) normal stress at the crack location

Fig. 6 Elastic behavior of the undamaged cell: theory vs. numerical results.

\subsection{Elastic behavior of the cracked cell}

Let us now consider the case of an elastic cracked inclusion, with neither debonding nor sliding between inclusion and matrix, as illustrated by Figure 7. This case is also purely elastic, but is unilateral, due to the presence of the transgranular crack. The compressive response is identical to that of the undamaged cell (see Section 4.1). In the tensile case, the crack induces highly non homogeneous fields for which no analytical solution exists to the authors' knowledge.

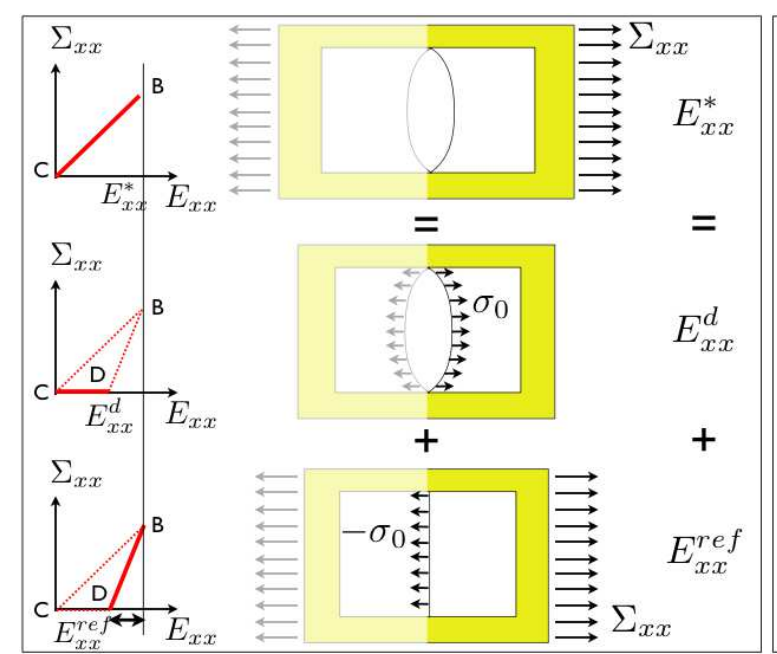

(a) virtual paths

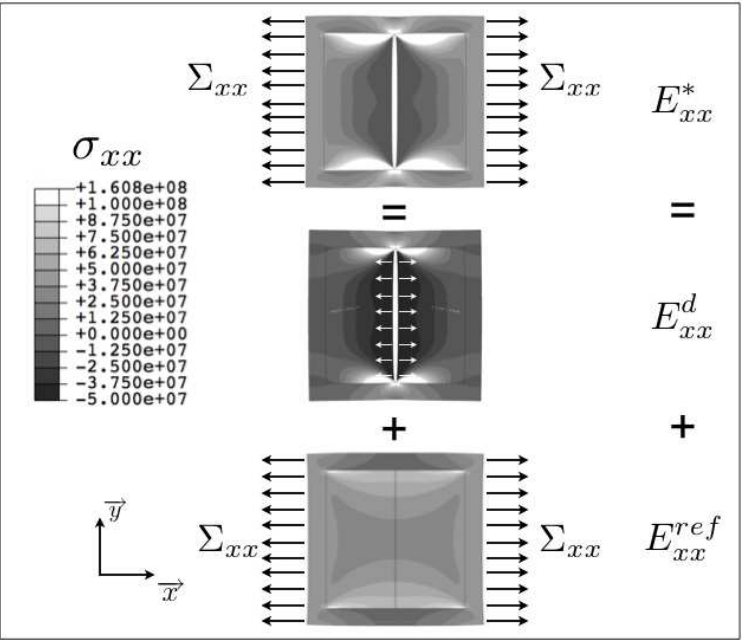

(b) numerical results

Fig. 7 Elastic behavior of the damaged cell: theory vs. numerical results.

This simple case will be employed to test the virtual path method in the presence of strong gradients. For this purpose, let us consider path CD-DB of Figure 7. Along CD, no external stress is applied, whereas an internal stress $\sigma_{0}$ is applied normal to the crack faces, in order that an overall strain $E_{x x}^{d}$ results. During DB, the crack displacement jump is maintained constant, while an external stress $\Sigma_{x x}$ is applied to the cell. The real stress state $\mathbf{B}$ is reached when the condition of free surfaces is met on the (open) crack mouth i.e., when the stress vanishes at this location. From Equation (29), this implies that

$$
\sigma_{0}=-\frac{K_{I}}{K_{x x}^{\prime}} \Sigma_{x x}
$$


For the first virtual path $\mathbf{C D}$, it is checked numerically that $\sigma_{0}$ is proportional to $E_{x x}^{d}$, and that the elastic energy is proportional to $\left(E_{x x}^{d}\right)^{2}$

$$
\begin{gathered}
E_{x x}^{d}=5.49 \times 10^{-10} \sigma_{0} \\
W^{d}=2.4 \times 10^{-10} \sigma_{0}^{2}
\end{gathered}
$$

where the numerical constants are expressed in suitable units. For the second virtual path DB, since the crack faces are held fixed, the cell stiffness is taken to be equal to that of the uncracked one. Equation (29) is then used to determine $\Sigma_{x x}$, whereas Equations (28) and (30) are used to evaluate the corresponding elastic strain $E_{x x}^{r e f}$ and energy $W^{r e f}$ respectively. According to the results of Section 2 and Ref. [1]), the strain $E_{x x}^{\star}$ and energy $W^{\star}$ at point $\mathbf{B}$ are given by

$$
\begin{aligned}
& E_{x x}^{\star}=E_{x x}^{r e f}+E_{x x}^{d} \\
& W^{\star}=W^{r e f}+W^{d}
\end{aligned}
$$

The resulting overall behavior can then be summarized in the following classical form [13]

$$
\begin{array}{r}
\Sigma_{x x}=K_{x x}^{e q}(1-\delta) E_{x x}^{\star} \\
W^{\star}=\frac{1}{2} K_{x x}^{e q}(1-\delta)\left(E_{x x}^{\star}\right)^{2}
\end{array}
$$

in which the damage parameter $\delta$ must be identified numerically in a straightforward fashion. In the present case, one obtains $\delta=0.69$, which corresponds to a softened modulus of $K_{x x}^{e q}(1-\delta)=1.03 \mathrm{GPa}$. The results are displayed in Figure 8, and compare favorably to numerical results. The quality of the match is of the same order as in the uncracked case, with a difference in strain energy of order $10 \%$, which appears satisfactory, considering the crack-induced gradients illustrated in Figure 7(b). The main drawback of the method is the need for numerical identification of the cracked cell stiffness (32), (33), which has to be performed for each cell geometry and material parameters.

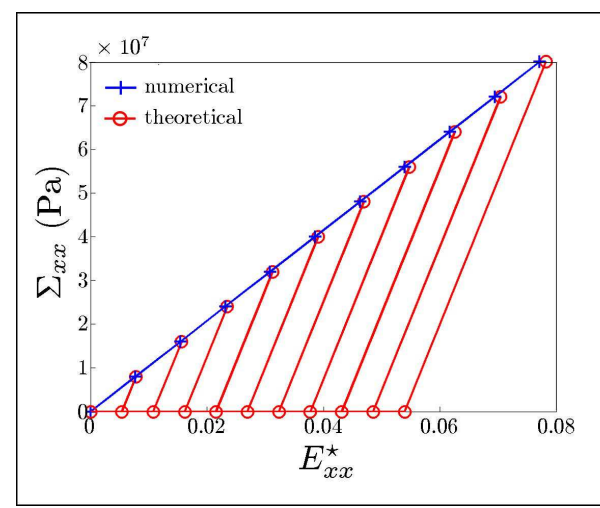

(a) mechanical response

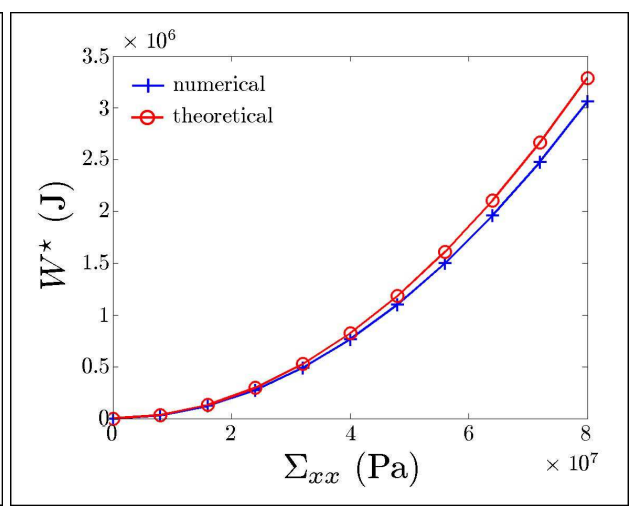

(b) energetic response

Fig. 8 Elastic behavior for the damaged cell: theory vs. numerical results.

\subsection{Inelastic behavior of the cracked cell: microfields}

The full behavior of the cracked cell can now be sought with some confidence. In the preceding sections, no confining lateral stress was applied to the cell, since linear elasticity was only considered. In the present case, however, where friction is to be accounted for, a lateral confining stress $\Sigma_{y y}$ is applied in addition to the longitudinal one $\Sigma_{x x}$, as depicted in Figure 9.

The virtual path is described in Figure 10. The first step OD consists in prescribing the inelastic displacement jump across the transgranular crack mouth, by applying an internal stress $\sigma_{0}$ on the crack mouth, without 


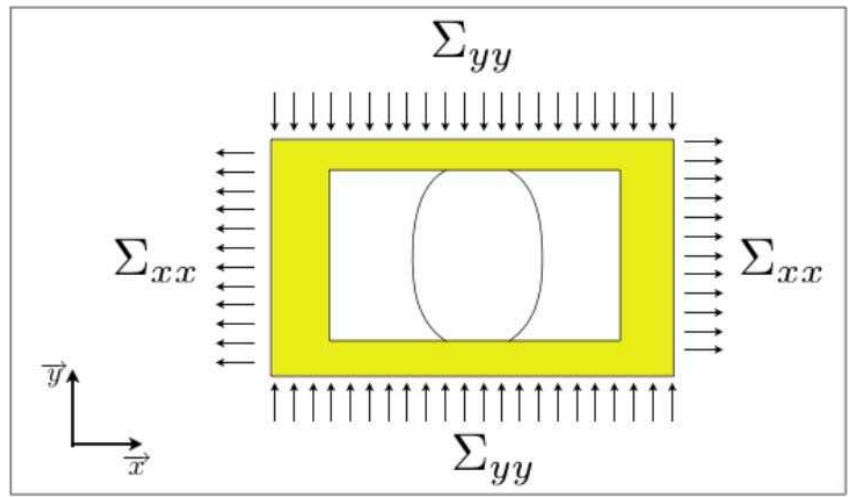

Fig. 9 Boundary conditions.

any external stress $\Sigma_{x x}=0$. Along this path, the shear stress grows at the inclusion-matrix interface, until Coulomb's condition is reached. At this time, a debond crack is assumed to initiate and grow, and friction occurs. The local fields will be built as follows. The lateral local stress is assumed as being uniform in the

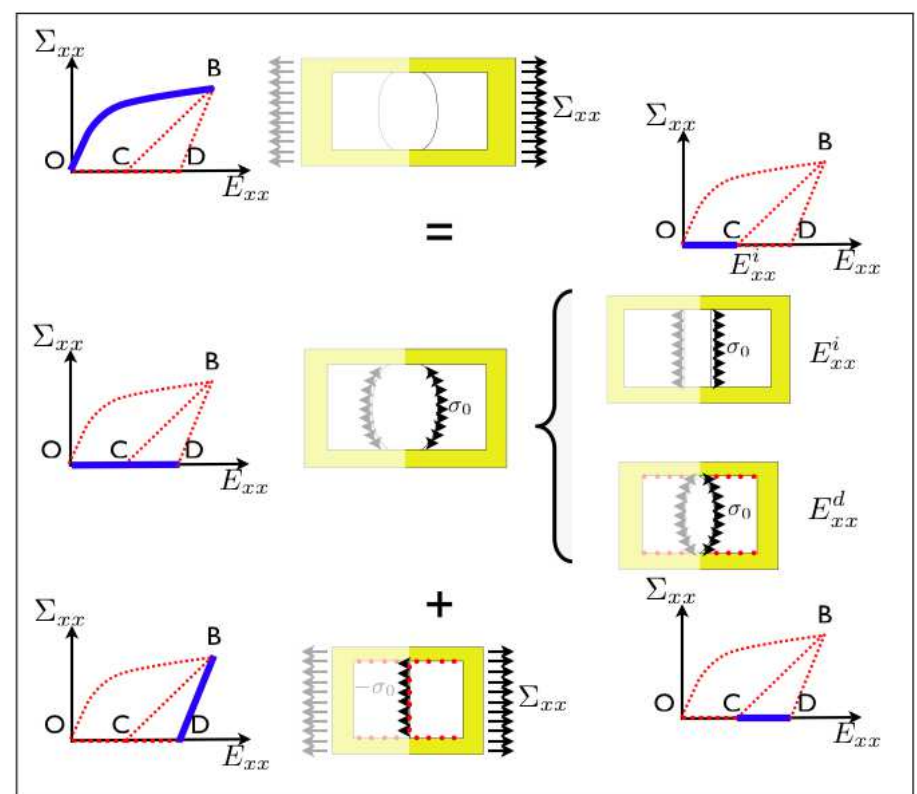

(a) the three stages of the virtual path

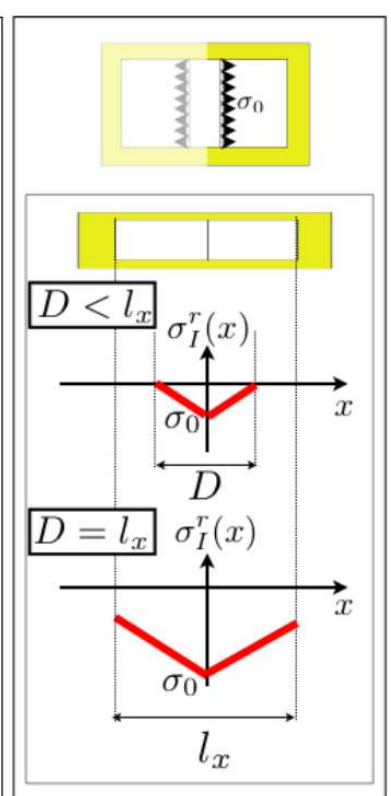

(b) Two cases occur, namely, the debond crack is growing or not

Fig. 10 The full modelling strategy.

inclusion. A suitable approximation for the inclusion-matrix interface stress $\tau$ is (see Figure 10(a))

$$
\tau=f \Sigma_{y y}
$$

where $f$ is the inclusion-matrix friction coefficient. The equilibrium of a slice of thickness $d x$ of the inclusion reads (see Figure 10(b))

$$
S_{I x} \sigma_{I x}^{i}(x+d x)-S_{I x} \sigma_{I x}^{i}(x)=-2 \tau d x \ell_{z}
$$

where $S_{I x}=\ell_{y} \ell_{z}$. This differential equation is solved

$$
\sigma_{I x}^{i}(x)=\sigma_{0}+p_{I} x
$$




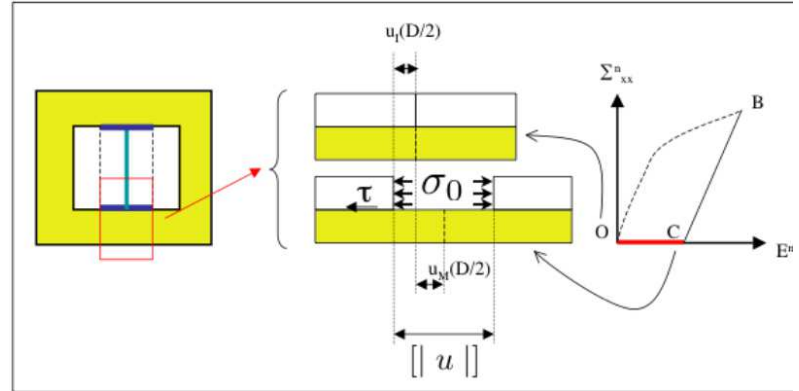

(a) internal detail of the damaged cell along the path OC

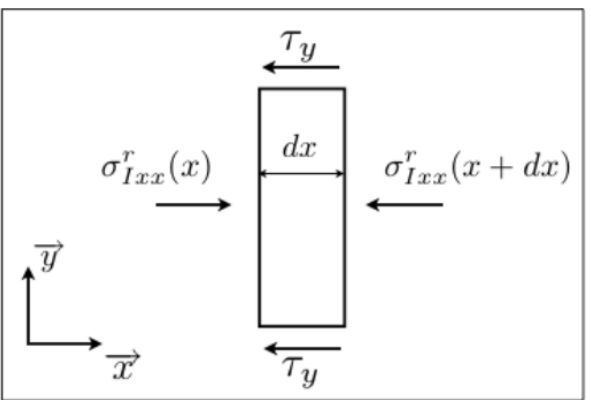

(b) equilibrium of a slice of the inclusion

Fig. 11 The inelastic virtual path.

where use was made of the condition $\sigma_{I x}^{i}(0)=\sigma_{0}$, and where

$$
p_{I}=-\frac{2 \tau \ell_{z}}{S_{I x}}
$$

The longitudinal stress field in the matrix is obtained by considering the equilibrium of slices of thickness $d x$ of the whole cell

$$
S_{I x} \sigma_{I x}^{i}(x)+S_{M x} \sigma_{M x}^{i}(x)=0
$$

where $S_{M x}=\left(L-\ell_{y}\right) \ell_{z}$. The longitudinal matrix stress is then

$$
\sigma_{M x}^{i}(x)=-\frac{S_{I x}}{S_{M x}} \sigma_{I x}^{i}(x)
$$

Let the length of the debond crack be denoted by $D$. Two cases must be distinguished, whether debonding is partial, i.e. $D<\ell_{x}$, or not, i.e. $D=\ell_{x}$.

4.4 Inelastic behavior of the cracked cell: Partial debonding

In the case of partial debonding, i.e. $D<\ell_{x}$, and since no external stress is applied, the local longitudinal stress field vanishes at $x=D / 2$, so that

$$
D=-2 \frac{\sigma_{0}}{p_{I}}
$$

The internal stress fields are given by Equations (40) and (43) for $x<D / 2$, and vanish elsewhere. For the virtual elastic path DB, during which the transgranular crack mouth is held fixed, the external stress $\Sigma_{x x}$ is applied, and generates uniform micro-stress fields $\sigma_{I x}^{r e f}(x)$ and $\sigma_{M x}^{r e f}(x)$, while Equation (31) provides the missing relationship between $\sigma_{0}$ and the applied external stress $\Sigma_{x}$. The overall inelastic strain is given by

$$
E_{x x}^{i}=2 \frac{u_{M}\left(\frac{D}{2}\right)}{L}=2 \frac{\int_{0}^{\frac{D}{2}} \epsilon_{M x}^{i}(x) d x}{L}=\frac{\tau \ell_{z} D^{2}}{2 K_{M} S_{M x} L}
$$

For monotonic tensile loading, Equation (44) applies, and is combined with Equation (31) to yield

$$
\Sigma_{x x}=\frac{K_{x x}^{\prime}}{d} E_{x x}^{i}
$$

where $d$ is given by

$$
d=\frac{K_{x x}^{\prime} S_{I x} D}{K_{M} S_{M x} L}
$$

Inserting Equation (45) into (46) yields

$$
E_{x x}^{i}=\frac{1}{2} \frac{K_{I}^{2} S_{I x}^{2}}{K_{M} S_{M x} L \ell_{z} \tau K_{x x}^{\prime 2}} \Sigma_{x x}^{2}
$$


This expression displays a stress-inelastic strain relationship of parabolic character, as illustrated in Figure 12, and further discussed below. The stored elastic energy is obtained using Equation (19). After some algebraic manipulations, it takes the following form $[6,14]$

$$
W^{i}=\frac{2}{3} \frac{K_{x x}^{\prime}\left(E_{x x}^{i}\right)^{2}}{d}
$$

The overall free energy then takes the following form

$$
\Psi=\frac{1}{2} K_{x x}^{e q}(1-\delta)\left(E_{x x}-E_{x x}^{i}\right)^{2}+\frac{2}{3} \frac{K_{x x}^{\prime}\left(E_{x x}^{i}\right)^{2}}{d}
$$

In this expression, the transgranular-induced reduction of longitudinal stiffness is accounted for through parameter $\delta$, obtained by comparing the initial stiffness (see Equation (28)) and the slope of Figure 8(a). The free energy $\Psi$ depends upon the observable variable $E_{x x}$ and two additional internal variables, namely $E_{x x}^{i}$ and $d$ respectively. The first one is analogous to the well-known plastic strain, and may increase or decrease depending upon external loading. Variable $d$, on the contrary, cannot decrease. In this respect, it constitutes a damage variable that describes the amount of internal surface involved in debonding.

The thermodynamic affinities are given by

$$
\begin{aligned}
X=\frac{\partial \Psi}{\partial E_{x x}^{i}} & =-K_{x x}^{e q}(1-\delta)\left(E_{x x}-E_{x x}^{i}\right)+\frac{4}{3} \frac{K_{x x}^{\prime} E_{x x}^{i}}{d} \\
& =-\Sigma_{x x}+\frac{4}{3} \frac{K_{x x}^{\prime} E_{x x}^{i}}{d}
\end{aligned}
$$

and

$$
y=\frac{\partial \Psi}{\partial d}=-\frac{2}{3} \frac{K_{x x}^{\prime}\left(E_{x x}^{i}\right)^{2}}{d^{2}}
$$

The dissipation $\mathcal{D}=-X \dot{E}_{x x}^{i}-y \dot{d}$ is then integrated along a monotonic tensile stress path, to yield the dissipated energy

$$
W^{d}=\frac{2}{3} \frac{K_{x x}^{\prime}\left(E_{x x}^{i}\right)^{2}}{d}
$$

Finally, the differential and integral Taylor-Quinney coefficients are respectively

$$
\begin{gathered}
\beta^{d}=\frac{\mathcal{D}}{\mathcal{D}+\dot{W}^{i}}=\frac{1}{2} \\
\beta^{\text {int }}=\frac{W^{d}}{W^{d}+W^{i}}=\frac{1}{2}
\end{gathered}
$$

This is a remarkable result. During continuous growth of the debonding crack, one half of the "inelastic" work is dissipated, and the other half is stored.This is only the case as long as debonding goes on, as shown in next section. This result is related to the particular situation studied above and is not general [14].

\subsection{Inelastic behavior of the cracked cell: Saturation}

When the length of the debond cracks reaches the inclusion length, saturation occurs. The variable $d$ reaches its maximum value $\widehat{d}$

$$
\widehat{d}=\frac{K_{x x}^{\prime} S_{I x} \ell_{x}}{K_{M} S_{M x} L}
$$

Besides, the overall inelastic strain $E_{x x}^{i}$ reaches a value $\widehat{E}_{x x}^{i}$ given by

$$
\widehat{E}_{x x}^{i}=\frac{\tau l_{z} l_{x}^{2}}{2 K_{M} S_{M x} L}
$$


whereas the longitudinal stress in the inclusion reads

$$
\widehat{\sigma}_{I x}^{i}(x)=-4 K_{M} \frac{S_{M x}}{S_{I x}} \frac{L}{\ell_{x}^{2}} \widehat{E}_{x x}^{i}\left(x-\frac{\ell_{x}}{2}\right)
$$

For continued loading, Equation (44) is no longer valid. However, the internal stress $\sigma_{0}$ must increase during the virtual loading path. Since the friction stress $\tau$ only depends on the confining stress $\Sigma_{y y}$, it does not vary, and neither does the longitudinal gradient of the local stress $\sigma_{I x}^{i}$. In that case, the local fields vary as illustrated in Figure 10. Hence, the price to pay for keeping a quasi-unidimensional stress field is a stress jump at the longitudinal inclusion matrix interface, i.e. at $x=\ell_{x} / 2$; however, global equilibrium is preserved. The local stresses are thus decomposed as

$$
\sigma_{I x}^{i}(x)=\widehat{\sigma}_{I x}^{i}(x)+\widetilde{\sigma}_{I x}^{i}(x)
$$

where $\widehat{\sigma}_{I x}^{i}(x)$ is given by Equation (58). Equation (39) is still valid, and so is Equation (40). The term $\widetilde{\sigma}_{I x}^{i}(x)$ thus represents the uniform part of the longitudinal stresses in the inclusion, and the following equation holds

$$
\widetilde{\sigma}_{I x}^{i}(x)=-\frac{K_{M} S_{M x} L}{S_{I x} \ell_{x}} \widetilde{E}_{x x}^{i}
$$

Using Equation (31), the stress-inelastic strain relationship under monotonic tensile loading reads

$$
\Sigma_{x x}=\frac{K_{x x}^{\prime}}{\widehat{d}}\left(E_{x x}^{i}+\widehat{E_{x x}^{i}}\right)
$$

This expression shows that the inelastic response is no longer parabolic, but becomes linear, as illustrated in Figure 12.

The stored energy can be calculated using Equations (19), (59) and (43), and takes the following form

$$
W^{i}=\frac{1}{6} \frac{K_{x x}^{\prime}}{\widehat{d}}\left[4\left(\widehat{E_{x x}^{i}}\right)^{2}-6 \widehat{E_{x x}^{i}}\left(E_{x x}^{i}+\widehat{E_{x x}^{i}}\right)+3\left(E_{x x}^{i}+\widehat{E_{x x}^{i}}\right)^{2}\right]
$$

The total free energy reads

$$
\Psi=\frac{1}{2} K_{x x}^{e q}(1-\delta)\left(E_{x x}-E_{x x}^{i}\right)^{2}+\frac{1}{6} \frac{K_{x x}^{\prime}}{\widehat{d}}\left[4\left(\widehat{E_{x x}^{i}}\right)^{2}-6 \widehat{E_{x x}^{i}}\left(E_{x x}^{i}+\widehat{E_{x x}^{i}}\right)+3\left(E_{x x}^{i}+\widehat{E_{x x}^{i}}\right)^{2}\right]
$$

The thermodynamic affinity of $E_{x x}^{i}$ is given by

$$
\begin{aligned}
X=\frac{\partial \Psi}{\partial E_{x x}^{i}} & =-K_{x x}^{e q}(1-\delta)\left(E_{x x}-E_{x x}^{i}\right)+\frac{K_{x x}^{\prime}\left(E_{x x}^{i}+\widehat{E_{x x}^{i}}\right)}{\widehat{d}} \\
& =-\Sigma_{x x}+\frac{K_{x x}^{\prime}\left(E_{x x}^{i}+\widehat{E_{x x}^{i}}\right)}{\widehat{d}}
\end{aligned}
$$

and the dissipation reads

$$
\mathcal{D}=-X \dot{E_{x x}^{i}}=\frac{K_{x x}^{\prime}}{\widehat{d}} \widehat{E_{x x}^{i}} E_{x x}^{i}
$$

The differential Taylor-Quinney coefficient becomes

$$
\beta^{d}=\frac{\mathcal{D}}{\mathcal{D}+\dot{W}^{i}}=\frac{\widehat{E_{x x}^{i}}}{E_{x x}^{i}+\widehat{E_{x x}^{i}}}
$$

Integrating the dissipation along a monotonic tensile stress path, the dissipated energy takes the following form

$$
W^{d}=\frac{K_{x x}^{\prime}}{\widehat{d}} \widehat{E_{x x}^{i}}\left(E_{x x}^{i}-\frac{1}{3} \widehat{E_{x x}^{i}}\right)
$$

and the integral Taylor-Quinney coefficient reads

$$
\beta^{i n t}=\frac{W^{d}}{W^{i}+W^{d}}=\frac{\widehat{E_{x x}^{i}}\left(E_{x x}^{i}-\frac{1}{3} \widehat{E_{x x}^{i}}\right)}{\frac{1}{2}\left(E_{x x}^{i}+\widehat{E_{x x}^{i}}\right)^{2}-\frac{2}{3}\left(\widehat{E_{x x}^{i}}\right)^{2}}
$$

These equations only account for longitudinal terms, and are thus only valid for constant confining stresses. The additional term accounting for the elastic energy of this lateral stress in the free energy was omitted for the sake of clarity. 
4.6 Comparison between theory and numerical simulations

Figures 12 and 13 compare this model with numerical results obtained in the same conditions, for a confining stress of $100 \mathrm{MPa}$. The comparison appears satisfactory. The qualitative response is captured correctly, and the difference is of the order of about $15 \%$, even up to $20 \%$ longitudinal strain. Given the crudeness of the quasi-unidimensional description of local fields, it would not be reasonable to expect a better result. The mismatch is essentially due to two-dimensional effects, and especially to the relatively high gradients in the neighborhood of the transgranular crack. In particular, the internal stress $\sigma_{0}$ is considered as uniform in the theory; this produces a curvature of the crack mouth, not accounted for in the theory. Figures 14 and 15 illustrate this fact, by comparing the above-mentioned simulations with complementary ones in which the transgranular crack faces are maintained artificially flat. In the latter case, the virtual path is controlled via the average of the internal stress $\sigma_{0}$ over the crack surface.

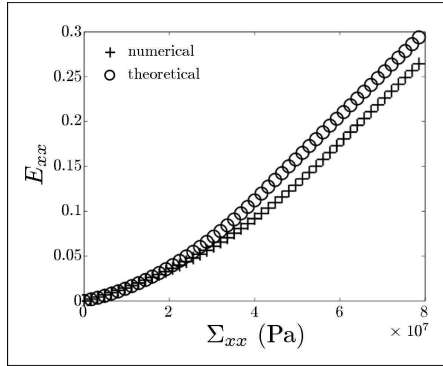

(a) total strain

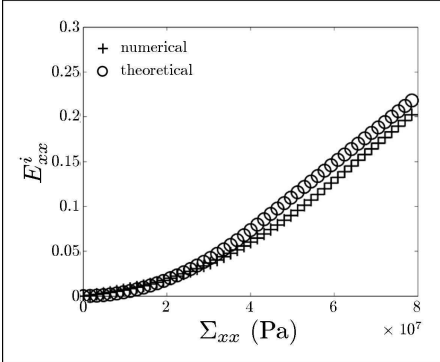

(b) inelastic strain

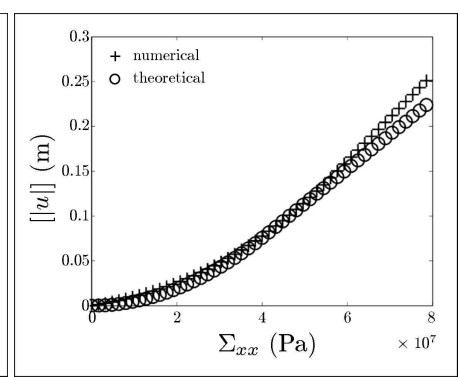

(c) displacement jump

Fig. 12 Comparison between theoretical and numerical results - Mechanical response

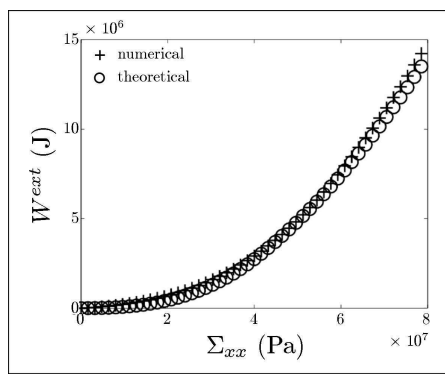

(a) external work

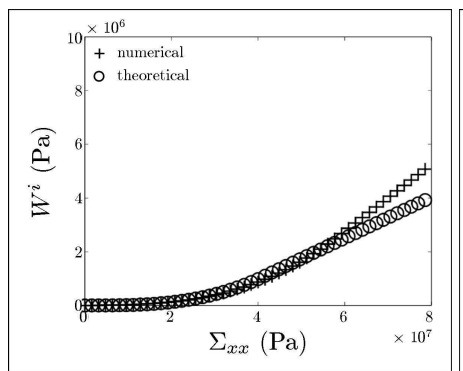

(d) stored energy

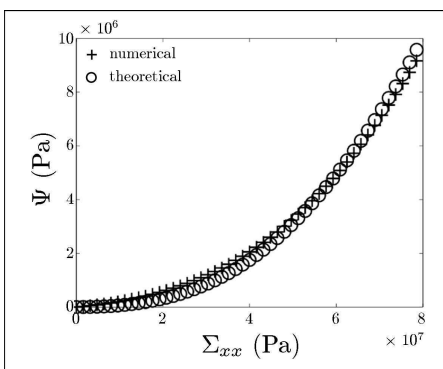

(b) free energy

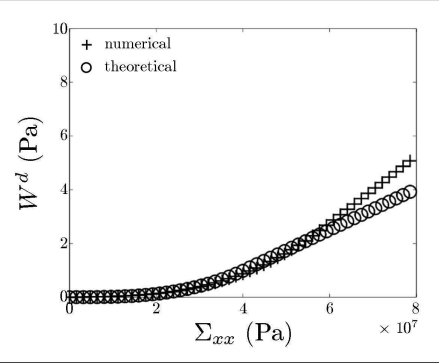

(e) dissipated energy

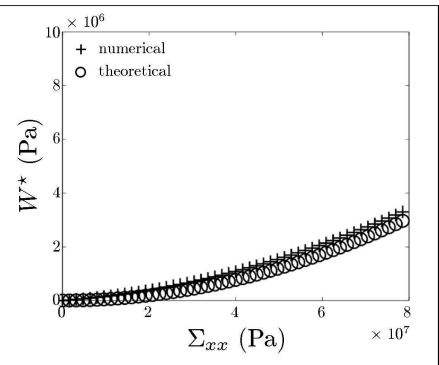

(c) recoverable elastic energy

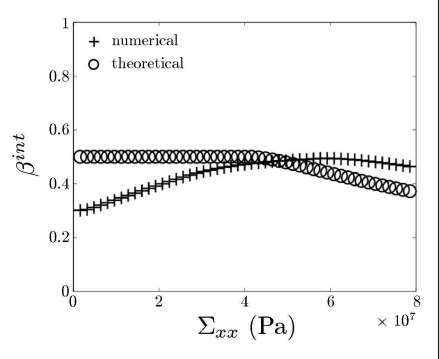

(f) integral Taylor-Quinney coefficient

Fig. 13 Comparison between theoretical and numerical results - Energetic response 


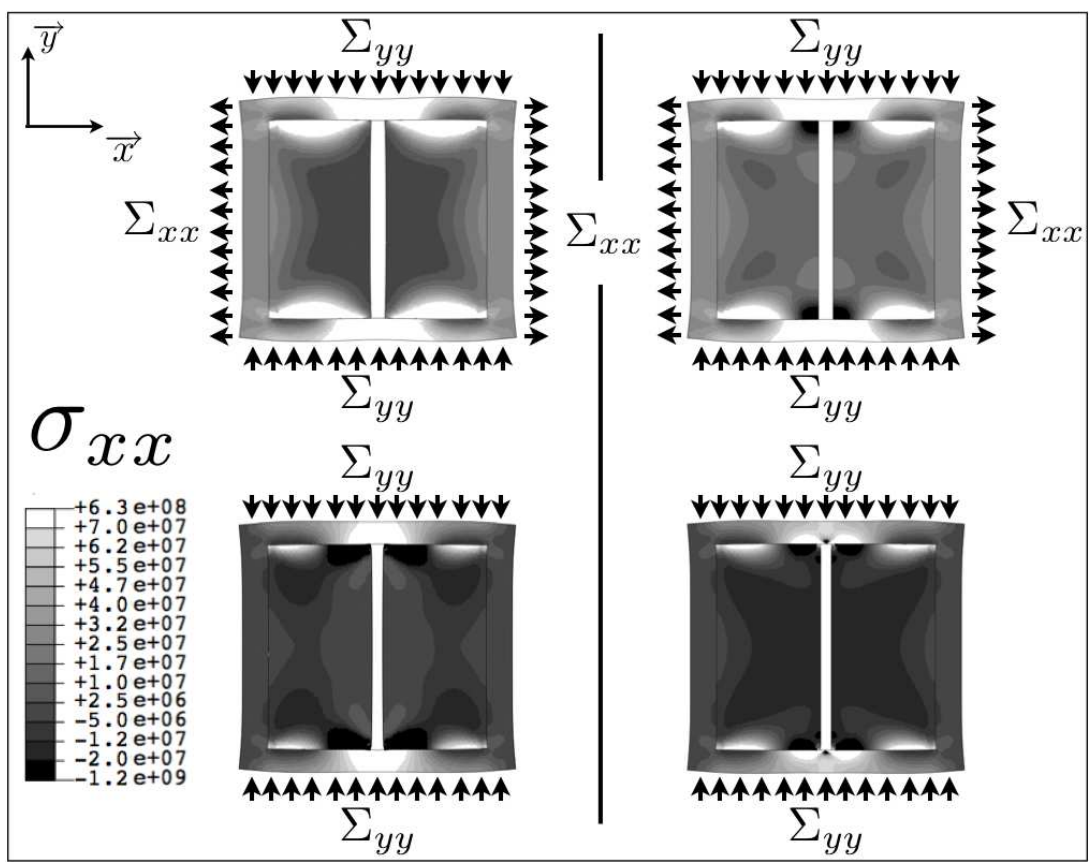

Fig. 14 Numerical illustration of the two-dimensional effects on the accuracy of the theory (longitudinal stress) - left: "normal" result; right: simulation with rigid crack faces; top: loaded stress state; bottom: virtually unloaded stress state.

\section{Displacement-driven approach}

\subsection{Introduction}

Figures 14 and 15 show that two-dimensional effects may have dramatic consequences for a highly heterogeneous elementary cell. It is thus likely that stress and displacement driven cells respond in a different manner,

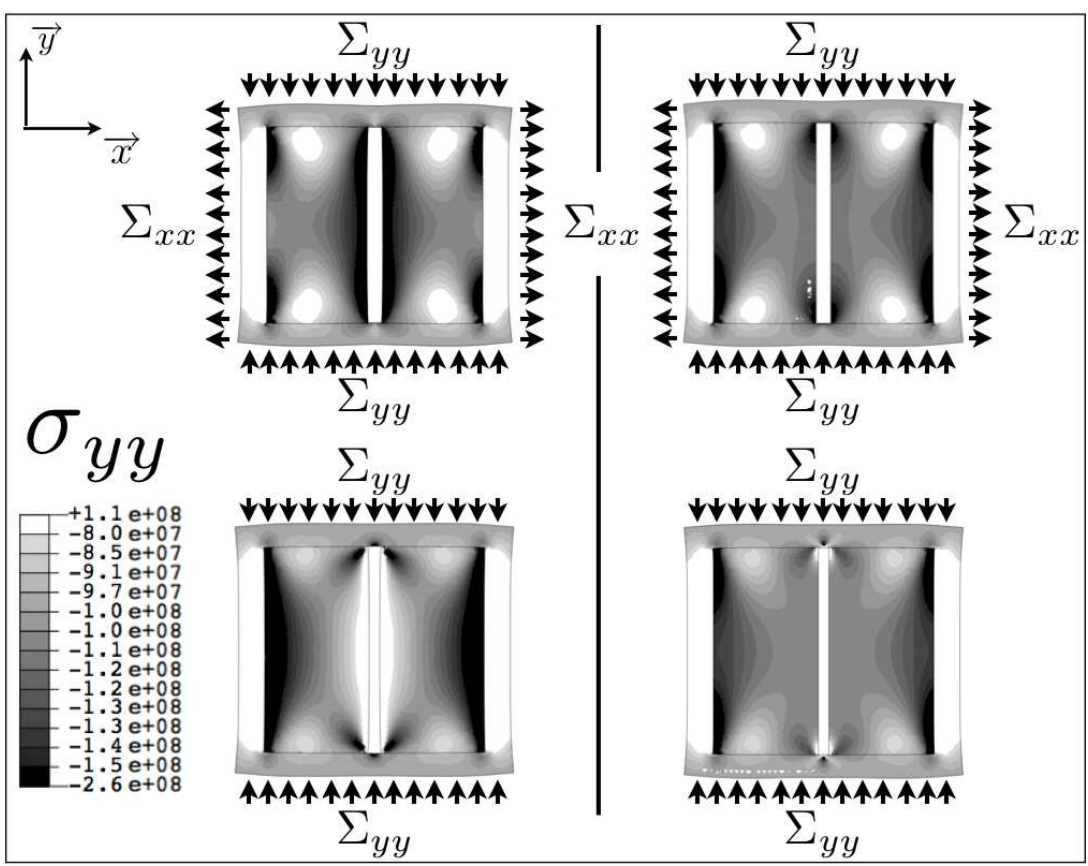

Fig. 15 Numerical illustration of the two-dimensional effects on the accuracy of the theory (transverse stress) - left: "normal" result; right: simulation with rigid crack faces; top: loaded stress state; bottom: virtually unloaded stress state. 
and in particular that prescribing a uniform displacement on cell boundaries result in overall stiffening. Although the stress-based approach is, on a computational standpoint, the best suited one for the present purpose, investigating the displacement-based approach might thus be appealing for future works on cell assemblies.

In that case, a constant strain $E_{y y}$ is first applied to the lateral surface of the cell, the longitudinal one remaining fixed. Hence, by macroscopic Poisson effect, the cell is under longitudinal compression. If a longitudinal tensile strain $E_{x x}$ is then applied to the axial surface of the cell, the compressive stress acting on the transgranular crack mouth must first be removed before friction starts. Moreover, Poisson's effect produces a lateral stress variation during axial straining. The process is less simple than in the stress-based approach, and will be sought in a more approximate manner.

Concerning analytical expressions, uniform strains are applied to the cell boundaries, the local stress and strain fields keep the same forms as in the stress-driven approach. Macroscopic stresses are given by Equations (2) and (7). In other words, the relationships between local and overall fields remain the same, and the stress and strain decompositions as well.

\subsection{Elastic response}

In a first step, the elastic response of the virgin cell is investigated. Using Equations (21), (22) and (23), the result is straightforward, and compared to numerical simulations in Figure 16, for the case of a lateral compressive strain applied at constant rate up to $E_{y y}=-0.1$, then maintained constant while a longitudinal tensile strain is applied at constant rate up to $E_{x x}=0.1$. In a second step, the elastic response of the cracked

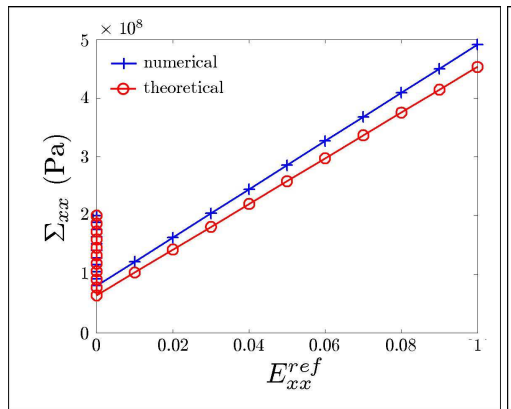

(a) Stress-strain response in the $x$ direction.

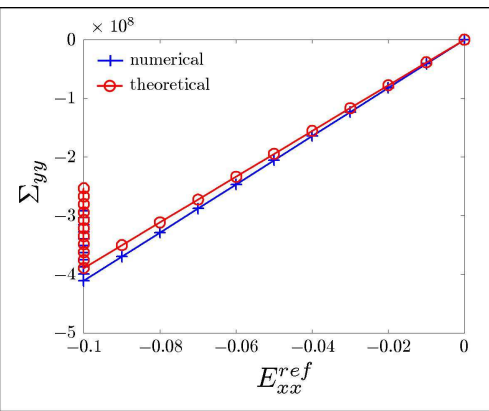

tion.

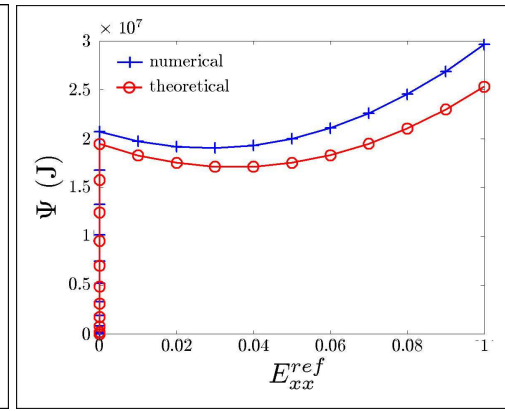

(c) Free energy.

Fig. 16 Displacement-based approach on the virgin cell: theoretical vs. numerical results.

cell is sought. This is performed in the same way as in the stress-based approach, i.e. by defining a degraded stiffness tensor in the following form

$$
\mathbf{E}^{\star}=\mathbf{S}^{\mathrm{d}} \boldsymbol{\Sigma}
$$

with

$$
\begin{gathered}
S_{x x}^{d}=\frac{1}{K_{x x}^{e q}(1-\xi)} ; S_{y y}^{d}=\frac{1}{K_{y y}^{e q}} ; S_{z z}^{d}=\frac{1}{K_{z z}^{e q}} \\
S_{x y}^{d}=\frac{-\nu_{x y}}{K_{x x}^{e q}} ; S_{x z}^{d}=\frac{-\nu_{z x}}{K_{z z}^{e q}} ; S_{y z}^{d}=\frac{-\nu_{z y}}{K_{z z}^{e q}}
\end{gathered}
$$

This form shows that the presence of the transgranular crack is assumed to have an effect on the longitudinal stiffness only. Hence, the degraded longitudinal compliance $S_{x x}^{d}$ is evaluated numerically, by identifying the constant $\xi$, under tensile loading, but without any confinement. Here, the damage parameter $\xi$ is taken to be different from $\delta$ of the stress-based approach, as it is to be identified numerically, and thus can be slightly different due to two-dimensional effects. The loading history is the applied in three steps, according to Figure 17. From the free state labelled 0 , the lateral strain $E_{y y}$ is applied up to point 1 . The lateral displacement is then frozen, and the longitudinal strain $E_{x x}$ applied. The response is that of the virgin cell up to point 2, where crack opening occurs. This is controlled through Equation (29). Finally, continued loading results in a softened response, up to point 3. 


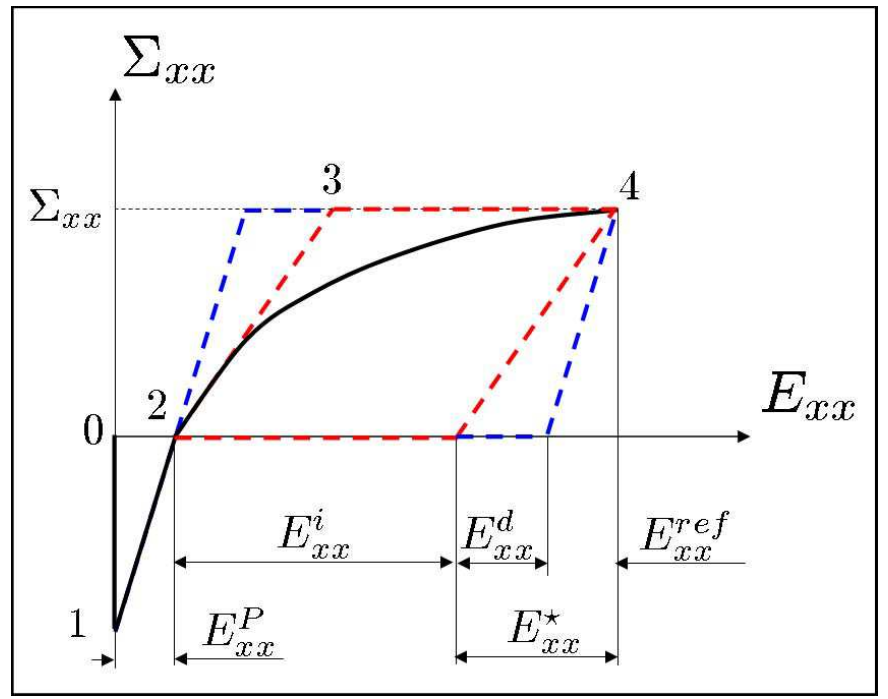

Fig. 17 Displacement-based approach on the damaged cell: loading phases, strain components and virtual elastic paths.

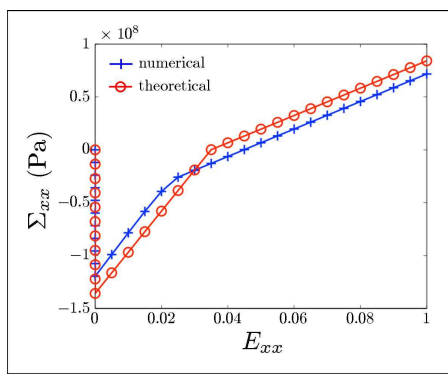

(a) Longitudinal response.

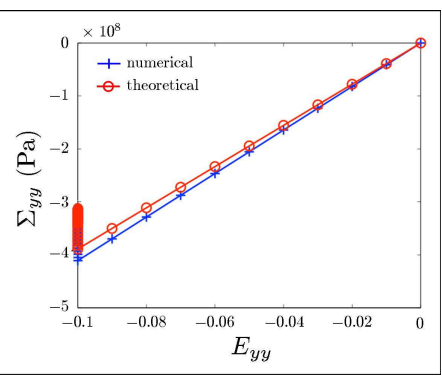

(b) Transverse response.

Fig. 18 Displacement-based approach on the damaged elastic cell: theoretical vs. numerical results.

The theoretical predictions are compared with numerical simulations in Figure 18. It is seen that separation of the crack faces is predicted later in the theoretical approach than in the simulations, this again being due to two-dimensional effects. The tensile phase begins with a decrease of the free energy, which corresponds to a decrease of lateral stresses. The effect is more pronounced in the theoretical results, but the correct trend is captured by the model.

\subsection{Inelastic response}

The main difficulty for describing debond crack friction lies here in the fact that, due to Poisson's effect, the interfacial stress is not constant during the tensile path. This problem can be overcome in a numerical approach, but again, a fully analytical approach is preferred. Hence, an additional assumption is needed, as will be explained now. This is illustrated in Figure 17.

As previously, two cases are to be distinguished, whether the inclusion-matrix crack is partially or totally debonded. In the first case, Equations (38) to (48) remain valid. The longitudinal overall strain $E_{x x}$ is split into three terms

$$
E_{x x}=E_{x x}^{P}+E_{x x}^{\star}+E_{x x}^{i}
$$

In this expression, $E_{x x}^{P}$ is the tensile overall strain needed to reach zero normal stress on the transgranular crack mouth (i.e. to overcome Poisson's effect), $E_{x x}^{\star}$ and $E_{x x}^{i}$ have the same signification as previously. The 
first term $E_{x x}^{P}$ is easy to find out, as shown in Section 5.2. The second term $E_{x x}^{\star}$ is given by

$$
E_{x x}^{\star}=\frac{\Sigma_{x x}}{K_{x x}^{e q}(1-\xi)}
$$

whereas the last term $E_{x x}^{i}$ is given by

$$
E_{x x}^{i}=-\frac{\tau_{y} \ell_{z} D^{2}}{2 K_{M} S_{M x} L}
$$

where, as previously

$$
\begin{gathered}
D=-2 \frac{\sigma_{0}}{p_{I}} \\
p_{I}=-\frac{2 \ell_{z}}{S_{I x}} \\
\tau_{y}=f_{I M} \Sigma_{y y} \\
\sigma_{0}=-\frac{K_{I}}{K_{x x}^{\prime}} \Sigma_{x x}
\end{gathered}
$$

Combining Equations (71), (72), (73) and (74) yields

$$
-\left(\frac{K_{I} S_{I x}}{K_{x x}^{\prime}}\right)^{2} \frac{\Sigma_{x x}^{2}}{2 K_{M} S_{M x} L \ell_{z} f_{I M}} \Sigma_{y y}+\frac{\Sigma_{x x}}{K_{x x}^{e q}(1-\xi)}-\left(E_{x x}-E_{x x}^{P}\right)=0
$$

This constitutes a second order equation in $\Sigma_{x x}$, easy to solve as soon as $\Sigma_{y y}$ is known. However, due to Poisson's effect, the latter evolves during tensile loading, and Equation (78) should be solved incrementally. In order to keep a closed-form solution, the assumption will be made that the lateral overall stress varies only slightly during extension, and sticks to its value at point 2 , labelled $\widehat{\Sigma}_{y y}$ in the following. The solution to Equation (78) with this additional assumption is

$$
\Sigma_{x x}=\frac{-\frac{1}{K_{x x}^{e q}(1-\xi)}+\sqrt{\left(\frac{1}{K_{x x}^{e q}(1-\xi)}\right)^{2}+4 \frac{C}{\hat{\Sigma}_{y y}}\left(E_{x x}-E_{x x}^{P}\right)}}{2 \frac{C}{\hat{\Sigma}_{y y}}}
$$

in which

$$
C=-\left(\frac{K_{I} S_{I x}}{K_{x x}^{\prime}}\right)^{2} \frac{1}{2 K_{M} S_{M x}} L \ell_{z} f_{I M}
$$

In the case of totally debonded matrix-inclusion crack, the solution is

$$
\Sigma_{x x}=\frac{E_{x x}-E_{x x}^{P}+\frac{\ell_{z} f_{I M} \widehat{\Sigma}_{y y} l_{x}^{2}}{2 K_{M} S_{M x} L}}{\frac{d_{M}}{K_{x x}^{\prime}}+\frac{K_{1}^{e}}{K_{x x}^{e}(1-\xi)}}
$$

This model is compared to numerical results in Figures 19 and 20. Again, despite the relative crudeness of the model assumptions, it captures correctly the main features of the elementary cell response.

\section{Conclusion}

The theory developed in a companion paper [1] to compute dissipated and stored energies in heterogeneous materials is used here to derive the first part of an ignition model for quasi-brittle energetic materials. An elementary cell is defined to be as simple as possible, although remaining representative of the microstructure of the class of materials at stake. This cell is made of a cracked hard elastic inclusion embedded in a homogeneous elastic soft matrix.

To get closed-form solutions, simplifying assumptions are made regarding the local mechanical fields. A fully analytical model is then built using the stress-based effective moduli approach, in the case of confined tension along the crack normal direction. Debonding and frictional sliding occur between the inclusion and the matrix. Using the concept of virtual elastic unloading, stored and dissipated energies are derived, and the integral Taylor-Quinney coefficient is shown to decrease slowly from an initial value of 0.5 . This means that 


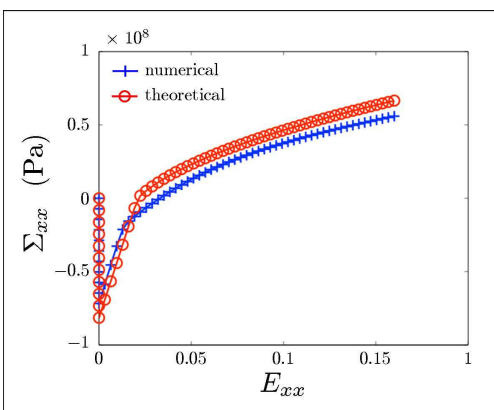

(a) Longitudinal stress-strain response.

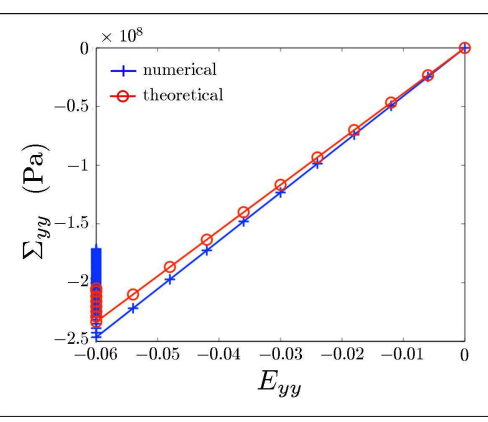

(b) Transverse stress-strain response.

Fig. 19 Comparison between displacement-driven theoretical and numerical results - Mechanical response.

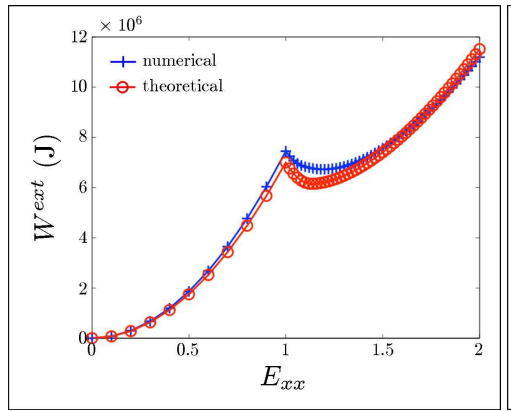

(a) External work.

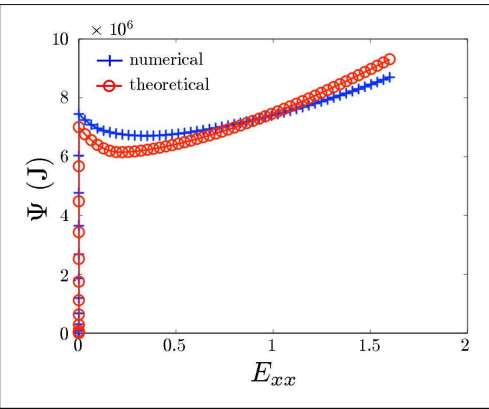

(b) Free energy.

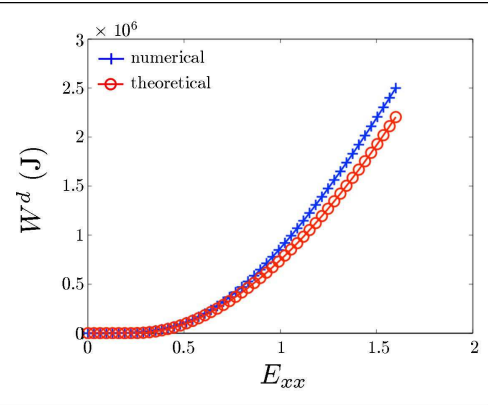

(c) Dissipated energy.

Fig. 20 Comparison between displacement driven theoretical and numerical results - Energetic response.

a large amount of energy is stored during the loading process, and could possibly be released and dissipated during unloading and reverse frictional sliding. Regarding ignition, this appears as a key point, which should be examined in the future.

Given the microstructure of the materials considered herein, in particular the high volume fraction of inclusions, it is likely that an assembly of such elementary cells appears much stiffer if displacement boundary conditions are applied to the cells. Therefore, although much less suited than the stress-based one, the strain-based approach was also investigated. This required additional assumptions, but it was shown that the approach can be handled. As a result, both approaches, when compared to numerical simulations using the finite element code ABAQUS, revealed reasonably accurate and, most of all, displayed the right trends.

The class of materials considered might involve dissipative behavior of the matrix. This could occur depending on the nature of the matrix itself, but also, for the material displayed in Figure 1, if a pressure (or strain-rate) induced glass transition takes place. In that case, the model presented here should be modified to account for, say, an elastic-plastic matrix. This can a priori be handled by the theory used herein [1]. Besides, the inclusion-matrix frictional sliding is not the only potential source of ignition. Many inclusions are cracked in the real material, thus providing additional potential heating sources for ignition. This new physical process can only take place under confined shear loadings, and must involve a completely separate modelling approach, especially regarding the representation of local fields. This will be the subject of a forthcoming paper.

\section{Acknowledgments}

This work was funded by a CEA grant. 


\section{References}

1. Vivier, G., Trumel, H., Hild, F., 2009. On the stored and dissipated energies in heterogeneous rate-independent systems: theory and simple examples. Continuum Mech. Thermodyn., 20, 411-427.

2. Vivier, G., 2008. Modélisation de l'allumage mécanique d'explosifs comprimés sous impacts modérés : apports de la thermodynamique des milieux continus. Ph. D. Thesis, Ecole Normale Supérieure de Cachan, France.

3. Bowden F. P., Yoffe A. F., 1952. Initiation and growth of explosion in liquids and solids, Cambridge University Press, Cambridge, UK.

4. Field J. E., Swallowe G. M., Heavens S. M., 1992. Ignition mechanisms of explosives during mechanical deformation, Proc. R. Soc. Lond. A, 382, 231-244.

5. Dienes J. K., 1996. A unified theory of flow, hot spots, and fragmentation with an application to explosive sensitivity, [in] High Pressure Shock Compression of Solids II (L. Davison ed.).

6. Andrieux S., Bamberger Y, Marigo J.-J., 1986. Un modèle de matériau microfissuré pour les bétons et les roches, J. Méc. Théor. Appl., 5, 471-513.

7. Hill R., 1967. The essential structure of constitutive laws for metal composites and polycrystals. J. Mech. Phys. Solids, 15, 79-95.

8. Mandel, J., 1964. Contribution théorique à l'étude de l'écrouissage et des lois de l'écoulement plastique. In: Becker, E., (ed.) Proc. 11th Int. Cong. Appl. Mech. Springer, Berlin, RFA, pp. 502-509.

9. Trumel, H., Lambert, P., Belmas, R., 2010. Mesoscopic investigations of the deformation and initiation mechanisms of a HMX-based pressed composition. Proc. 14th Int. Symp. on Detonation, Coeur d'Alene (ID), USA.

10. Taylor G. I., Quinney H., 1934. The latent energy remaining in a metal after cold working, Proc. R. Soc. Lond. A, 143, 307-326.

11. Aravas N., Kim K.-S., Leckie F. A., 1990. On the Calculations of the Stored Energy of Cold Work, J. Eng. Mat. Tech., 112, 465-470.

12. Lemaitre J., Chaboche J.-L., 1990. Mechanics of Solid Materials, Cambridge University Press, Cambridge (UK).

13. Lemaitre J.,1992. A Course on Damage Mechanics, Springer-Verlag, Berlin (Germany).

14. Hild F., Burr A., Leckie F. A., 1996. Matrix Cracking and Debonding in Ceramic-Matrix Composites, Int. J. Solids Struct., 33, 1209-1220. 